\title{
9 MAPEAMENTO DOS RECURSOS HUMANOS E SERVIÇOS OFERTADOS POR DOIS CENTROS DE ATENÇÃO PSICOSSOCIAL INFANTO-JUVENIL
}

\author{
| Claudia Moreira de Lima'; Sue Ellen Ferreira Modesto Rey de Figueiredo²; Akeisa Diele Ribeiro Dalla Vechia ${ }^{3}$ |
}

\section{RESUMO}

OBJETIVO: Este estudo teve como objetivo caracterizar e identificar os serviços disponíveis à crianças e adolescentes com variados tipos de transtornos mentais atendidas em dois Centros de Atenção Psicossociais Infanto-Juvenis (CAPSi) do estado de Mato Grosso, Brasil.

MÉTODOS: Foi aplicado um questionário semiestruturado auto respondido pelos coordenadores dos CAPSi participantes da pesquisa entre os meses de janeiro e fevereiro de 2018 e foram utilizadas fichas para o registro das observações feitas pelos pesquisadores nas unidades. Os principais serviços disponíveis às crianças/adolescentes que frequentam os respectivos CAPSi foram: acolhimento, atendimento por equipe multidisciplinar (médicos, enfermeiros, psicólogos, terapeutas ocupacionais, fonoaudiólogos e assistentes sociais), oficina/grupo terapêutico, reuniões da equipe técnica, visita domiciliar, atendimento individual aos usuários e seus familiares, participação em assembleias e comemoração de datas festivas/mês.

RESULTADOS: Os resultados apontam que a equipe atuante nos serviços busca desenvolver ações de forma interdisciplinares idealizando um cuidado de forma integral, apesar da falta de recursos humanos adequados.

CONCLUSÕES: Conhecer a situação da assistência ao público infanto-juvenil com algum transtorno de saúde mental pode ser um primeiro passo para a sensibilização de líderes das áreas da saúde e da educação para novas propostas de assistência a estes indivíduos bem como seus familiares.

\section{PALAVRAS-CHAVE: Saúde mental; Crianças; Adolescentes; Transtornos mentais}

\section{RESUMEN}

"Asignación de recursos humanos y servicios ofrecidos por dos centros de atención psicosocial para niños y adolescentes"

OBJETIVO: Este estudio pretende caracterizar e identificar los servicios disponibles para los niños y adolescentes con diversos tipos de trastornos mentales conocidos en dos centros de atención psicosociales para los niños (Centro (CAPSi)) en el Estado de Mato Grosso, Brasil.

METODOLOGIA: Un cuestionario semiestructurado fue contestado por los coordinadores de la encuesta del Centro (CAPSi) entre los meses de enero y febrero de 2018 e hicieron fichas para las observaciones formuladas por los investigadores en las unidades. Los principales servicios disponibles para niños y adolescentes que asisten a su centro (CAPSi) eran: acogerlos, cuidado por un equipo multidisciplinario (médicos, enfermeras, psicólogos, terapeutas ocupacionales, audiólogos y trabajadores sociales), taller/grupo terapéutico, reuniones de personal técnico, visita a domicilio, asistencia individual a los usuarios y sus familias, participación en reuniones y conmemoración de fechas festivas/mes.

RESULTADOS: Los resultados señalan que el equipo que actúa en los servicios busca desarrollar acciones de manera interdisciplinaria, idealizando un cuidado de forma integral, a pesar de la falta de recursos humanos adecuados.

CONCLUSIONES: Para conocer la situación de la asistencia a los niños y adolescentes con un trastorno de salud mental pueden ser un primer paso hacia la sensibilización de líderes en las áreas de salud y la educación a nuevas propuestas de ayuda a estas personas, así como su familia.

DESCRIPTORES: Salud mental; Niños; Adolescentes; Trastornos mentales

\begin{abstract}
"Human resources mapping and offered services by two childjuvenile psychosocial attention centers"

AIM: This study's objective is to characterize and identify the avaliable services to children and teenagers with various types of mental disorders attended at two Child-Juvenile Psychosocial Attention Centers(CJPAC) from the state of Mato Grosso, Brazil.

METHODS: It was applied a semi-structured questionnaire which was self answered by the coordinators from the reaserch participating CJPACs between the months of January 2018 and Febuary 2018 forms were used to record the observations made by the researchers at the units. The main avaliable services to the children/teenagers that attend the respective CJPAC were: hosting, multidisciplinary team treatment (doctors, nurses, psychologist, occupational therapists, speech therapists, social assitants), therapeutic group/workshop, technical team meetings, home vist, individual user attendance and their relatives, assembly participation and celebration of festive dates/months.

RESULTS: The results point towards that the active team on these services seek to develop actions in an interdisciplinary way idealizing them with integral caution, although the lack of adequate human resources.

CONCLUSIONS: To know the situation of public child-juvenile assitance services to it's public with any mental heath disorder can be the step to the sensibilization of leaders of health and education areas to new assistance proposals of such individuals and their relatives as well.
\end{abstract}

\section{KEYWORDS: Mental health; Children; Teenagers; Mental disorders}

Submetido em 31-03-2018

Aceite em 19-07-2018

\footnotetext{
1 Enfermeira Especialista em Urgência e Emergência; Mestranda na Universidade de Cuiabá, Programa Ambiente e Saúde, Brasil, cml_claudiamoreira@hotmail.com

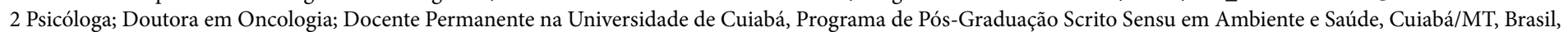
suemodesto@hotmail.com

3 Enfermeira; Mestranda na Universidade Federal do Estado de Mato Grosso, 78300-000 Tangará da Serra/MT, Brasil, akeisa_drdv@hotmail.com
}

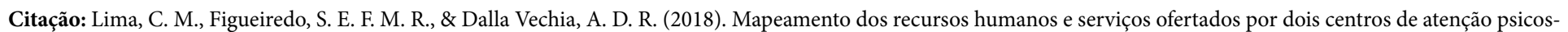
social infanto-juvenil. Revista Portuguesa de Enfermagem de Saúde Mental (20), 65-71. doi: 10.19131/rpesm.0228 


\section{INTRODUÇÃO}

Nos últimos anos os transtornos mentais na população infanto-juvenil representam um sério problema de Saúde Pública, sendo responsáveis por agravantes na qualidade de vida, podendo causar incapacidades, morbidades e até morte prematura (Paula, Miranda e Bordin, 2010). Estudos apontam para uma prevalência de $10 \%$ a $20 \%$ de crianças e adolescentes do mundo sofram de algum transtorno mental de leve a grave (Organização Mundial da Saúde, 2003). A nível nacional essa taxa varia de acordo com a abordagem do estudo, ficando em $12 \%$ a $24,6 \%$ em estudos realizados utilizando instrumentos de rastreamento ou de $7 \%$ a $12,7 \%$ em estudos desenvolvidos por entrevistas (Paula et al., 2014).

Entretanto apesar da premente temática a nível mundial a atenção à saúde mental de crianças e adolescentes passou a fazer parte da agenda das políticas públicas tardiamente (Delfine e Reis, 2012). No Brasil a Lei Federal 10.216 em 2001, trata da Reforma Psiquiátrica e através da publicação da portaria 336/02 que se consolidou o modelo assistencial dos Centros de Atenção Psicossociais Infanto-Juvenis (CAPSi) como um importante dispositivo de atendimento à saúde mental do público infanto-juvenil, tendo como objetivo o cuidar com foco na compreensão da subjetividade de maneira mais humanizado e menos hospitalocêntrico (Carvalho, Gondim, Holanda e Alencar, 2014). Nesse contexto os CAPSi são responsáveis por atender crianças e adolescentes com idade compreendida entre zero a 18 anos que apresentem algum transtorno psíquico (Carvalho et al., 2014). De acordo com os dados de agosto de 2017 havia no Brasil 2.465 CAPS implantados, sendo 1.191 CAPS I, 505 CAPS II, 100 CAPS III, 324 CAPSad, 106 CAPSad III e 229 CAPSi (Ministério da Saúde, 2017). Com relação aos CAPSi a região centro Oeste conta com 10 unidades, destes quatro estão no Estado de Mato Grosso, localizadas nos municípios de Cuiabá atual capital do Estado e os municípios de Várzea Grande, Rondonópolis e Cáceres cada município com 1 unidade (Secretaria de Estado de Saúde de Mato Grosso, 2017). Há uma escassez de trabalhos que tratam da assistência em saúde mental direcionada ao público infanto-juvenil, dessa forma, almejamos com este estudo fornecer conhecimento acerca da assistência no campo da saúde mental da criança e adolescente, agregando dados aos estudos existentes, para isso nosso estudo buscou conhecer e descrever os serviços prestados por dois Centros de Atenção Psicossocial Infanto-Juvenil (CAPSi) do Estado de Mato Grosso.

\section{MÉTODOS}

Trata-se de um estudo do tipo transversal, descritivo realizado em dois Centros de Atenção Psicossocial Infanto-juvenil (CAPSi) localizados nos municípios de Cuiabá e Várzea Grande, estado de Mato Grosso - Brasil. A escolha das unidades foi por conveniência.

A coleta das informações ocorreu entre os meses de janeiro e fevereiro de 2018. Para as entrevistas com os coordenadores de cada CAPSi participante do estudo, utilizou-se roteiro semiestruturado autorrespondido, que versou sobre os atendimentos ofertados durante o ano de 2017, abordando itens relacionados a funcionalidade das unidades bem como informações acerca da equipe profissional atuante no respectivo ano. Utilizaram-se fichas para o registro das observações feitas pelos pesquisadores nas unidades.

Posteriormente, os dados coletados pelo questionário foram codificados e armazenados em planilha eletrônica do programa Microsoft Excel ${ }^{\circledR}$ para posterior análise que ocorreu em três etapas: tabulação dos dados; análise estatística simples; triangulação com dados qualitativos e interpretação crítica dos dados em confronto com a literatura pesquisada. Em um primeiro momento foi descrito o serviço agrupando os dados dos dois questionários nas categorias serviços prestados e recursos humanos. A posteriori foram comparadas as categorias operacionais com informações disponíveis na literatura nacional e internacional acerca da temática e normas regulamentadas.

O diário de campo teve como objetivo detalhar fatos do cotidiano dos CAPSis servindo como complemento às análises anteriores, proporcionando acesso a realidade dos fatos, contribuindo na caracterização e discussão dos dados das entrevistas.

Esta pesquisa foi desenvolvida após aprovação pelo Comitê de Ética em Pesquisa da Universidade de Cuiabá-UNIC, sob o Parecer n. 2.421.481, CAAE: 80172417.8.0000.5165. A pesquisa foi desenvolvida sem financiamento. Todos os participantes assinaram o Termo de Consentimento Livre e Esclarecido (TCLE), por meio do qual foram informados sobre as medidas para assegurar o anonimato e a confidencialidade sobre a origem dos dados obtidos. O presente estudo obedeceu às normas de pesquisa com seres humanos, segundo a Resolução n 466/2012 do Conselho Nacional de Saúde. 


\section{RESULTADOS}

As unidades participantes deste estudo somam um quantitativo de 2.548 usuários cadastrados e em atendimento regular durante o ano de 2017, com idade compreendida entre 0 a 18 anos, sem um período estabelecido para acompanhamento/tratamento, este é realizado até a percepção da equipe da possibilidade de alta assistencial e/ou quando esta não acontece e o usuário completa a maior idade é feito encaminhamento ao Centro de Atenção Psicossocial (CAPS). As unidades prestam atendimento em dois períodos (matutino e vespertino) de segunda e sexta-feira, com cuidados a crianças e adolescentes com transtornos mentais severos e/ou persistente dentre eles: autismo, neurose grave, psicose, depressão, ideação e tentativa de suicídio, bipolaridade, transtorno opositor desafiador, quadros de déficit intelectual associado a outra transtorno mental e hiperativo associado a outro transtorno mental, sendo de maior recorrência atendimentos a casos de Autismo psicótico neurótico grave e tentativa e ideação ao suicídio.

Os serviços nas unidades são desenvolvidos por uma equipe multiprofissional atuando sob uma lógica interdisciplinar, onde os profissionais atuam de forma distinta, com competências próprias de sua formação ao mesmo tempo que realizam atividades em coletivo com os demais colegas desenvolvendo um trabalho interdisciplinar. As equipes das unidades somam um quantitativo de vinte e quatro profissionais, estes divididos entre unidade I e unidade II, estando estes profissionais em caráter efetivo $(28,57 \%$ e $60 \%)$ e provisório $(71,43 \%$ e 40\%) respectivamente. A composição das 'categorias profissionais' atuantes nas unidades engloba médicos, enfermeiros, psicólogos, terapeutas ocupacional, fonoaudiólogos, assistentes social e técnicos de enfermagem. A distribuição profissional em cada unidade de estudo pode ser melhor visualizada no quadro 1 .

Quadro 1 - Descrição do Número de Participantes da Pesquisa, Segundo a Quantidade por Unidade. Cuiabá, 2018.

\begin{tabular}{|l|l|l|l|l|}
\hline Categoria Profissional & \multicolumn{2}{l|}{$\begin{array}{l}\text { CAPSi Uni- } \\
\text { dade I }\end{array}$} & \multicolumn{2}{l|}{ CAPSi Unidade II } \\
\hline & No & $\%$ & No & $\%$ \\
\hline Médico & 01 & 7,14 & 01 & 10,0 \\
\hline Enfermeiro & 02 & 14,29 & 02 & 20,0 \\
\hline Psicólogo & 02 & 14,29 & 02 & 20,0 \\
\hline Terapeuta Ocupacional & 02 & 14,29 & 00 & 0,0 \\
\hline Fonoaudiólogo & 03 & 21,41 & 01 & 10,0 \\
\hline Assistente Social & 02 & 14,29 & 02 & 20,0 \\
\hline Técnico de Enfermagem & 02 & 14,29 & 02 & 20,0 \\
\hline Total & 14 & 100 & 10 & 100 \\
\hline
\end{tabular}

Fonte: CAPSi Cuiabá/MT, CAPSi Várzea Grande/MT. Coordenação de Saúde Mental-MT. 2018
As atividades realizadas pelos profissionais são especificas da abordagem da formação de cada profissional este atuando de forma interdisciplinar, além da realização de um trabalho organizado em forma de escala mensal, com a participação nas atividades por todos os profissionais. Estas ações estão detalhadas no Quadro 2.

Quadro 2 - Atividades Desenvolvidas pelo Profissional em Formato de Escala

\begin{tabular}{|l|}
\hline Acolhimento inicial \\
\hline Oficina/grupo terapêutico \\
\hline Participação em reunião da equipe técnica \\
\hline Visita domiciliar \\
\hline Atendimento individual aos usuários e aos familiares \\
\hline Participação na assembleia com os usuários \\
\hline Elaboração de datas festivas/mês \\
\hline
\end{tabular}

Dentre a atividades supracitadas, faz-se necessário destacar que o profissional médico, não compartilha das mesmas atividades, ficando destinado especificadamente aos atendimentos clínicos, visitas domiciliares e participação nas reuniões técnicas.

Foram investigados também os serviços ofertados à clientela e seus familiares, ressaltamos que nesta etapa da pesquisa por conta do instrumento de pesquisa utilizado para a coleta dos dados não foi possível identificar elementos mais qualitativos relacionados às formas de participação de usuários, familiares e profissionais.

$\mathrm{O}$ atendimento de crianças e adolescentes com algum problema de saúde mental bem como atenção a seus familiares, vem sendo desenvolvido nos CAPSi investigados, com ações que envolvem oficinais terapêuticas, atendimentos em grupos, atendimentos individuais, atividades comunitárias, visitas e atendimentos domiciliares. A assistência é ofertada com atendimentos diferenciados para cada grupo, com atividades e horários por faixa etária e transtorno, o que contribuiu para uma evolução quanto ao tratamento.

No que tange a articulação dos CAPSi com outros setores e movimentos sociais, são realidade das instituições, que mantem contato próximo com escolas, Unidades Básicas de Saúde, Conselho tutelar e promotoria/ justiça.

Quanto à assistência prestada ao usuário nos CAPS, a Portaria n. ${ }^{\circ}$ 336/2002 (p.4, 2002) prevê que: “[...] os pacientes assistidos em um turno (04 horas) receberão uma refeição diária; os assistidos em dois turnos (08 horas) receberão duas refeições diárias [...]".

A respeito desse aspecto, as unidades exploradas nesta pesquisa disponibilizam refeição aos usuários assistidos conforme as normas do MS supracitada. 
Outro dado significativo do estudo refere-se à 'Reunião de Equipe'. Todos os CAPSi participantes da pesquisa realizam reunião de equipe semanalmente viabilizando a discussão de casos, avaliação das atividades desenvolvidas, levantamento de problemas e propostas visando melhorar a prestação dos serviços.

\section{DISCUSSÃO}

Os Centros de Atenção Psicossocial Infanto-juvenil (CAPSi), foram uma importante conquista no que se refere ao cuidado desprendido à saúde de crianças e adolescentes com transtornos mentais, com estimativas que apontam para uma estatística de que uma entre quatro a cinco crianças e adolescentes no mundo apresentam algum tipo de transtorno mental (Patel, Flisher, Hetricks \& McCorry, 2007). Os dados encontrados neste estudo com relação ao perfil nosológico das unidades traz o suicídio (tentativa ou ideação) e o autismo ( $\mathrm{Au}$ tista psicótico neurótico grave) entre as causas de maior utilização dos serviços. Cediço, nos últimos anos, vem sendo divulgados dados alarmantes da ocorrência de autismo, está síndrome merece atenção especial, tendo em vista que estudos em todo o mundo apontam para uma prevalência de $1 \%$ da população infanto-juvenil, sendo 1 caso a cada 68 crianças (Untoiglich, 2013; Brasil, 2015). No que tange o suicídio, este é considerado um problema de saúde pública. Um estudo epidemiológico realizado em 101 países entre 2000 a 2009 sobre suicídio entre todas as faixas etárias, foi constatado que $14,7 \%$ dos suicídios abordados na pesquisa ocorreram em crianças e adolescentes (Kõlves \& De Leo, 2015). No que se refere aos profissionais atuantes nas unidades, estes são na sua maioria contratados, o que torna o cuidado frágil uma vez que com o interromper dos contratos alguns não atuam novamente na unidade, havendo assim a substituição por outro profissional. Não é nossa intenção desmerecer o profissional que assume a atividade, entretanto, destarte, essa rotatividade de pessoal acarreta dificuldade no estabelecimento de vínculo usuário-profissional-família, uma vez que esta interação quando possível ser estabelecida é um ponto positivo na construção da rede de atenção em saúde mental, tendo esse vínculo como um percursor de melhor prognóstico (Paes, Schimith, Barbosa e Righi, 2013; Ribeiro, 2015). Por outro lado, a diversidade de profissionais atuantes nas unidades para a realização dos cuidados demonstra a existência de uma prática multiprofissional, essa interação entre diferentes profissionais, possibilita uma articulação nas intervenções propostas.
Sendo essa diversidade profissional essencial nos centros de atenção psicossocial independente de sua classificação, visando atender o perfil dos usuários do serviço, tentando adequar-se à realidade sociocultural da região. Para tanto, os cuidados desprendidos são desenvolvidos a partir do Projeto Terapêutico Singular (PTS), que tem a construção da atividade envolvendo equipe, usuário e família (Boccardo, Zane, Rodrigues e Mãngia, 2011).

Diante ao exposto as equipes quando formadas por profissionais de categorias diversas devem levar em consideração os usuários que serão atendidos, de modo a possibilitar um acompanhamento contínuo além de uma melhor absorção da terapêutica por cada usuário (Anjos Filho e Souza, 2017), porem se faz necessário mencionar a observação de dificuldade na articulação entre profissionais em alguns momentos, o que é esperado tendo que a sua formação o leva a priorizar distintos aspectos no trabalho terapêutico.

Desta feita a equipe de um CAPS (esteja ele em qualquer categoria de classificação) deve ser contemplada com uma diversidade profissional, onde cada tipo de CAPS tem características próprias quanto aos tipos e quantidade de trabalhadores. No que tange a equipe profissional mínima para estruturação de um CAPSi temos: "01 (um) médico psiquiatra, ou neurologista ou pediatra com formação em saúde mental; b - 01 (um) enfermeiro. c - 04 (quatro) profissionais de nível superior entre as seguintes categorias profissionais: psicólogo, assistente social, enfermeiro, terapeuta ocupacional, fonoaudiólogo, pedagogo ou outro profissional necessário ao projeto terapêutico" (Portaria 336, 2002, p. 4-5). Com relação aos 'profissionais que compõem as equipes' é importante ressaltar que as duas unidades apresentam defasagem em recursos humanos em nível profissional conforme o preconizado pela Portaria

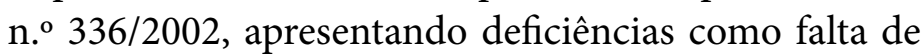
médico psiquiatra ou com formação em saúde mental. Em pesquisa realizada na cidade da Paraíba, também foi constatado a carência quanto a distribuição dos recursos humanos no serviço, em especial também na área médica (Clementino et al., 2016). Ainda, de modo a reforçar os dados apurados, destaca-se que quando o profissional não tem uma proximidade com a área de saúde mental o cuidado fica prejudicado, o que é confirmado no estudo realizado por Ronchi e Avellar (2013), onde os depoentes desconheciam a dinâmica de funcionamento do CAPSi, assim como a regulamentação dos serviços, o que levava a um atendimento precário. 
Há de se considerar além disso que a falta de uma equipe mínima atuante neste setor de saúde caracteriza-se como um fator desestabilizador para o serviço, tendo como ponto crítico problemas para a prática da integralidade, uma vez que a multiprofissionalidade conforme mencionado contribui para a introdução de novas formas de abordagem/tratamento (Paes et al.,2013). No que tange as atividades a serem desenvolvidas nestas unidades, o MS preconiza a realização de atividades de caráter individual e em grupos. Diante ao aludido, as duas unidades oferecem diferentes atividades as crianças e adolescentes, com atendimento tanto individual quanto coletivo, desenvolvem atividades por meio de oficinas/ grupos terapêuticos, realizam visitas domiciliares, além de atividades voltadas ao atendimento a família conforme necessidade identificada. Faz-se enfatizar que as unidades dispõem de horários diferenciados para cada faixa etária e transtorno tratado. Em relação a este achado, a literatura cientifica evidencia que a assistência adequada de acordo com o transtorno psíquico, faixa etária e um ambiente acolhedor facilitam o cuidado e a relação entre usuário e profissional, estes fundamentais para uma assistência humanizada (Ribeiro, 2015). A realização de atividades tanto no individual quanto no coletivo, assim como a entrega de materiais (lápis, borracha, giz de cera, papel sulfite, brinquedos entre outros), fornecidos durante o atendimento possibilita ao usuário identificar ações necessárias no seu dia a dia. Ações estas preconizadas pelo Ministério da Saúde (2010), onde trata da atenção dispensada ao usuário através da disponibilização de materiais que favorecem o vínculo e a criação de um ambiente adequado às suas necessidades. Outra atividade importante realizada com às crianças e os adolescentes são as oficinas de artes e de histórias, além da realização de grupos com adolescentes. As atividades não têm um padrão estabelecido, elas são desenvolvidas de acordo com o grupo formado ou usuário atendido, levando em consideração a individualidade de cada um. As visitas domiciliares são realizadas com o objetivo de promover momentos direcionados para o usuário e família. Esta abordagem permite acompanhar o usuário no seu individual, favorece a realização de atendimento assistencial e educativo além de possibilitar a inserção da família no tratamento o que gera um suporte para a continuidade deste. Por meio deste recurso é direcionado um suporte a família do usuário, que muitas vezes estão em situação de falta de suporte social, atividade ocupacional e a própria ausência de suporte familiar (Pereira, Cézar, Reisdorfer e Cardoso, 2014).
Ainda em relação aos serviços ofertados, uma das unidades informou oferecer visitas institucionais e matriciamento, além de realizar assembleia geral. No que diz respeito ao matriciamento este é uma abordagem satisfatória neste setor de saúde, tendo em vista que a atenção em saúde é feita de forma compartilhada com vistas à integralidade e à resolubilidade do cuidado, por meio do trabalho interdisciplinar. $\mathrm{O}$ matriciamento tem sido bem avaliado quando as equipes conseguem adequada interlocução, possibilitando estabelecer corresponsabilidade no tratamento dos usuários (Delfine e Reis, 2012). Os autores ainda enfatizam que as ações desenvolvidas extramuros são essenciais neste setor de saúde visando atender a diversidade de problemas que envolvem a saúde mental neste foco do público infantojuvenil, garantindo assim maior resolutividade em casos de sofrimento psíquico, porém, o que poderia ser usado a favor do tratamento - diferentes setores atuando juntos - mostra-se um desafio a ser superado, tendo que as intervenções em saúde mental a este público são desafiadoras, justificadas pela falta de parâmetro essencial durante o atendimento e pela complexidade da situação e também do ambiente.

No que diz respeito a rotina dos CAPSis, a oferta de lanche é tida como a finalização do trabalho em ambas as unidades estudadas. Mister identificar que esse processo não deve ser visto como um simples momento de refeição, mais sim um momento que deve levar em consideração as especificidades do usuário, onde o ambiente destinado à 'hora do lanche' é um ambiente que pode e deve ser tomado como um local de possibilidade de intervenção, mais uma vez evidenciado pelo Ministério da Saúde (Ministério da Saúde, 2010), onde traz que a atenção dispensada aos usuários, deve estar atenta aos espaços utilizados pelos pacientes de modo geral, assim todo e qualquer espaço pode ser agregado no atendimento vistas a uma terapêutica.

$\mathrm{Na}$ questão referente a 'reunião da equipe' sua realização permite uma maior integração e interação entre os profissionais, possibilitando melhor discussão dos casos atendidos. É um importante dispositivo neste setor de saúde que auxilia no (re)delinear do trabalho através das falas interdisciplinares dos casos apresentados pelos profissionais, essa interdisciplinaridade contribui para o surgimento de conteúdo de inovação em saúde e traz um olhar diferente para os procedimentos que estão sendo abordados, identificando a necessidade de mudança de terapêutica, adequação ou complementação (Anjos Filho e Souza, 2017). 


\section{CONCLUSÕES}

Os principais serviços disponíveis às crianças/adolescentes que frequentam os respectivos CAPSi deste estudo foram: acolhimento, atendimento por equipe multidisciplinar (médicos, enfermeiros, psicólogos, terapeutas ocupacionais, fonoaudiólogos e assistentes sociais), oficina/grupo terapêutico, reuniões da equipe técnica, visita domiciliar, atendimento individual aos usuários e seus familiares, participação em assembleias e comemoração de datas festivas/mês.

$\mathrm{Na}$ pesquisa empreendida, pôde-se verificar potencialidades dos serviços de saúde mental, onde os dados apurados permitem constatar que a equipe atuante nos serviços busca desenvolver ações de forma interdisciplinares idealizando um cuidado de forma integral, apesar da falta de recursos humanos adequados diante do preconizado.

Nesse enredo ressalta-se que que a não inclusão das outras instituições existentes no Estado evidencia uma limitação, destarte, nosso interesse é despertar e ampliar a discussão acerca da temática, onde os resultados desse estudo ajudam a conhecer um pouco mais a situação da assistência ao público infanto-juvenil com algum transtorno de saúde mental, o que pode ser um primeiro passo para a sensibilização de líderes das áreas da saúde e da educação para novas propostas de assistência a estes indivíduos bem como seus familiares.

\section{REFERÊNCIAS BIBLIOGRÁFICAS}

Anjos Filho, N. C., Souza, A. M. P. (2017). A percepção sobre o trabalho em equipe multiprofissional dos trabalhadores de um Centro de Atenção Psicossocial em Salvador, Bahia, Brasil. Interface - Comunicação, Saúde, Educação, 21 (60), 63-76. Doi: 10.1590/180757622015.0428

Boccardo, A. C. S., Zane, F. C., Rodrigues, S., Mângia, E.F. (2011). O projeto terapêutico singular como estratégia de organização do cuidado nos serviços de saúde mental. Revista de Terapia Ocupacional. 22 (1), 85-92. Doi: 10.11606/issn.2238-6149.v22i1p85-92

Brasil (2010). Núcleo Técnico da Política Nacional de Humanização. (4 ${ }^{\mathrm{a}}$ ed.) Brasília: Ministério da Saúde. Disponível em: http://bibliotecadigital.puc-campinas. edu.br/services/e-books/humanizasus_documento_ gestores_trabalhadores_sus.pdf
Carvalho, I. L., Gondim, A.P.S., Holanda, T. T., Alencar, V.P. (2014). CAPSi: Avanços e Desafios após uma Década de Funcionamento. Cadernos Brasileiros de Saúde Mental/Brazilian Journal of Mental Health, 6 (14), 4260.

Clementino, F. S., Miranda, F. A. N., Martiniano, C. S., Marcolino, E. C., Pessoa Junior, J. M., Dias, J. A. (2016). Avaliação de Estrutura Organizacional dos Centros de Atenção Psicossocial do Município de Campina Grande, Paraíba. Revista Brasileira de Ciências da Saúde, 4 (20), 261-268. Doi: 10.4034/RBCS.2016.20.04.01

Delfine, P. S. S e Reis, A. O. A. (2012). Articulação entre serviços públicos de saúde nos cuidados voltados à saúde mental infanto-juvenil. Cadernos de Saúde Pública, 28 (2), 357-366. Doi: 10.1590/S0102-311X2012000200014

Kõlves, K., De Leo, D. (2015). Child, Adolescent and Young Adult Suicides: A Comparison Based on the Queensland Suicide Registry. Journal of Child Adolescent Behavior, 3 (3), 1-5. Doi: 10.4172/23754494.1000209

Brasil. (2001). Lei no 10.216, de 6 de abril de 2001 - Dispõem sobre a proteção e os direitos das pessoas portadoras de transtorno mentais e redireciona o modelo assistencial em saúde. Disponível em: https://saudedireito.org/2014/05/26/lei-10-216-de-2001-reformapsiquiatica-e-os-direitos-das-pessoas-com-transtornos-mentais-no-brasil/

Ministério da Saúde (2015). Linha de cuidado para a atenção às pessoas com transtornos do espectro do autismo e suas famílias na Rede de Atenção Psicossocial do Sistema Único de Saúde. Disponível em: http://bvsms. saude.gov.br/bvs/publicacoes/linha_cuidado_atencao_ pessoas_transtorno.pdf

Ministério da Saúde (2017). Panorama e Diagnóstico da Política Nacional de Saúde Mental. Disponível em: http://portalarquivos2.saude.gov.br/images/pdf/2017/ setembro/04/2a-Apresentacao-CIT-Final.pdf

Paes, L. G., Schimith, M. D., Barbosa, T. M., Righi, L. B. (2013). Rede de atenção em saúde mental na perspectiva dos coordenadores de serviços de saúde. Trabalho, Educação e Saúde, 11 (2), 395-409. Doi: 10.1590/S198177462013000200008 
Patel V., Flisher, A. J., Hetricks., McCorry P. (2007). Mental health of young people: a global publichealth challenge. The Lancet, 369 (9569), 1302-1313. Doi:10.1016/ S0140-6736(07)60368-7

Paula, C. S, Bordin, I. A., Mari, J. J., Velasque, L., Rohde, L. A., Coutinho, E. S. (2014). The mental health care gap among children and adolescents: data from an epidemiological survey from four Brazilian regions. PLoS One, 9 (2),1-9. Doi: 10.1371/journal.pone.0088241

Paula, C. S., Miranda, C. T., Bordin, I. A. S. (2010). Saúde mental na infância e adolescência: revisão dos estudos epidemiológicos brasileiros. In O. Tanaka, L. Yoshimi, \& E. Ribeiro (Orgs.), Atenção em saúde mental para crianças e adolescentes no SUS (pp.75-92). São Paulo: Editora Hucitec.

Pereira, S. S., Cézar, J. G. S., Reisdorfer, E., Cardoso, L. (2014). Visita domiciliar aos pacientes portadores de transtorno mental: ampliando as opções terapêuticas possíveis em um serviço ambulatorial. Saúde \&Transformação Social, 5 (1),91-95. Disponível em: http:// incubadora.periodicos.ufsc.br/index.php/saudeetransformacao/article/view/2410/3656
Brasil. (2002). Portaria No 336/GM, 19 de fevereiro de 2002 - Construção de uma rede de Centros de Atenção Psicossocial Infanto-juvenis em território Nacional. Brasília: Ministério da Saúde.

Ribeiro, M. C. (2015). Psychosocial care center workers in Alagoas, Brazil: interstices of new practices. Interface - Comunicação, Saúde, Educação, 19 (52), 95-108. Doi: 10.1590/1807-57622014.0151

Ronchi, J. P., Avellar, L. Z. (2013) Ambiência na Atenção Psicossocial InfantoJuvenil: um estudo no CAPSi. Saúde Sociedade, (22) 4, 1045-1058. Disponível em: http://www.scielo.br/pdf/sausoc/v22n4/08.pdf

Secretaria de Estado de Saúde de Mato Grosso. (2018). Guia de Serviços Saúde Mental. Área Técnica de Saúde Mental/COAPRE/SAS/SES-MT. Disponível em: http:// www.saude.mt.gov.br/cidadao/345/guia-de-servico

Untoiglich, G. (2013). As oportunidades clínicas com crianças com sinais de autismo e seus pais. Estilos da Clínica, 18 (3), 543-558. Disponível em: http:// pepsic.bvsalud.org/scielo.php?script=sci_arttext\&pid $=$ S1415-71282013000300008

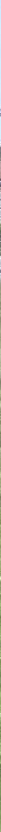

